\title{
Edge-functionalized and substitutional doped graphene nanoribbons: electronic and spin properties
}

\author{
F. Cervantes-Sodi, G. Csányi, S. Piscanec, A. C. Ferrari \\ Department of Engineering, University of Cambridge, Cambridge, UK
}

\begin{abstract}
Graphene nanoribbons are the counterpart of carbon nanotubes in graphene-based nanoelectronics. We investigate the electronic properties of chemically modified ribbons by means of density functional theory. We observe that chemical modifications of zigzag ribbons can break the spin degeneracy. This promotes the onset of a semiconducting-metal transition, or of an halfsemiconducting state, with the two spin channels having a different bandgap, or of a spin-polarized half-semiconducting state-where the spins in the valence and conduction bands are oppositely polarized. Edge functionalization of armchair ribbons gives electronic states a few $\mathrm{eV}$ away from the Fermi level, and does not significantly affect their bandgap. N and B produce different effects, depending on the position of the substitutional site. In particular, edge substitutions at low density do not significantly alter the bandgap, while bulk substitution promotes the onset of semiconductingmetal transitions. Pyridine-like defects induce a semiconducting-metal transition.
\end{abstract}

\section{INTRODUCTION}

Graphene is the latest carbon allotrope to be discovered [1, 2, 3, 4, 5], and it is now at the center of a significant experimental and theoretical research effort. In particular, near-ballistic transport at room temperature and high carrier mobilities (between 3000 and 100000 $\mathrm{cm}^{2} / \mathrm{Vs}$ ) [1, 6, 7] make it a potential material for nanoelectronics [8, 9, 10, 11], especially for high frequency applications.

It is now possible to produce graphene samples with areas exceeding thousands of square microns by means of micro-mechanical cleavage of graphite, and much larger by "expitaxial" growth on $\mathrm{SiC},[3,12,13,14,15,16,17]$. An ongoing effort is being devoted to large scale production and growth on different substrates of choice [18, 19, $20,21,22]$.

Graphene can be readily identified in terms of number and orientation of the layers by means of elastic and inelastic light scattering, such as Raman 23, 24, 25, 26, 27] and Rayleigh spectroscopies [28, 29]. Raman spectroscopy also allows monitoring of doping and defects [23, 25, 30, 31]. Once identified, graphene layers can be processed into nanoribbons by lithography $[8,11,16,32]$.

As for carbon nanotubes (CNTs) [33, 34], electron confinement modifies the electronic structure of graphene, when this is cut into nanoribbons (GNRs) [35, 36, 37, 38, 39, 40, 41]. When GNRs are cut from a single graphene layer, their edges could in general consist of a combination of regions having an armchair or a zigzag geometry [42, 43, 44, 45]. If a ribbon is uniquely limited by one of these edges, it is defined either as an armchair GNR (AGNR) or as a zigzag GNR (ZGNR) (See, e.g., Fig. 1) [35, 36, 45].

GNRs are the counterpart of nantubes in graphene nanoelectronics. Indeed, the confinement of the electronic wavefunctions and the presence of the edges open a bandgap, making them suitable for the realization of devices. Due to their potential technological applica- tions, their electronic structure has been widely investigated $\sqrt{35}, 36,37,38,39,40,41,446,47,48,49,50,51$, $52,53,54,55,56,57,58,59,660,61,62,63]$, with particular attention to the factors determining the presence and the size of the gap.

CNTs can be metallic or semiconducting, depending on their chirality. This could lead to a fully carbon based electronics, where semiconducting tubes are used as channels and metallic ones as interconnects [64, 65, 66]. However, the present lack of control on the chirality prevents to engineer their electronic properties on-demand, and is a major barrier to industrial implementation.

GNRs could combine the exceptional properties of graphene, and the possibility of being manufactured by means of industry-amenable large scale top down planar technologies, which would uniquely define their chirality.

Introducing impurities and functional groups can be an effective way to control electronic properties of GNRs. On the other hand, covalently bonded impurities are likely to be a by-product of the production processes.

Here, we investigate the effect of chemical disorder on the band structure of ZGNRs and AGNRs. We first study edge functionalization of ZGNRs with a set of different radicals, elucidating the role played (i) by the ribbons width, (ii) by the concentration of the functional groups along the ribbon's edges, and (iii) by the presence of one- and two-sided edge functionalization. We find that edge functionalization breaks the spin degeneracy, leading to four possible outcomes. In the first case, the ribbon maintains its semiconducting nature, and the top of the valence band and the bottom of the conduction band belong to the same spin channel. We refer to this as "spin-selective half-semiconductivity" [57]. In the second case, the ribbon is still semiconducting, but the top of the valence band and the bottom of the conduction band belong to opposite spin channels. We refer to this as as "spin-polarized half-semiconductivity" [67]. In the third and the fourth cases, the band gap of one or both spin channels closes. In the latter case, the ribbon undergoes a semiconductor-metal transition, while in the former, it 
behaves as a half-metal [50, 68]. Edge functionalization on AGNRs at the moderately low densities we studied has negligible effect on the gap.

We then consider chemical doping on H-terminated AGNR and ZGNR. AGNRs are always semiconducting, so, in principle, $\mathrm{B}, \mathrm{N}$, or $\mathrm{O}$ atomic substitutions can result in some form of electrical doping. However, given the particular nature of the ribbons, it is not clear a-priori whether chemical doping of AGNRs would result in the formation of defect states within the gap, like in carbon nanotubes [69, 70, 71, 72] and bulk semiconductors, or if it would rather cause a shift of the Fermi level similarly to what observed in graphene [23, 73, 74]. Thus, we investigate in detail the effects of atomic substitutions on the electronic structure of AGNRs. We find that upon B and $\mathrm{N}$ edge-substitution, impurity levels appear far away from the band gap, slightly renormalizing its width. On the contrary, $\mathrm{N}$ and $\mathrm{B}$ substitution in the bulk of the ribbons result in impurity levels near the gap, and in a shift of the Fermi energy within one of the continuum levels of the pristine ribbon. Atomic substitution in edge functionalized ZGNR and pyridine-like impurities in AGNR induce semiconductor-metal transitions for high impurity densities, but do not give impurity levels close to the top or bottom of the gap, unlike the case of nanotube doping.

\section{BACKGROUND}

\section{A. Band structure of GNRs}

GNRs can be thought as single wall CNTs cut along a line parallel to their axis and then unfolded into a planar geometry. Since on a graphene plane the zigzag and armchair directions are orthogonal, this procedure transforms an armchair CNT into a ZGNR and a zigzag CNT into an AGNR. However, this correspondence between the geometry of CNTs and GNRs is not entirely reflected in their electronic properties. Indeed, in contrast to CNTs, where armchair tubes are always metallic and zigzag could be metallic or semiconducting depending on the chiral angle [75], the earliest theoretical studies of the electronic structure predicted all ZGNR to be metallic, while AGNR were expected to be semiconducting [35, 36, 37, 38, 46, 47]. The metallic character of ZGNR was attributed to the presence of a high density of edge states at the Fermi energy [36, 37, 38, 46].

More recently, spin-polarized DFT calculations have found that the ground state of ZGNR has a antiferromagnetic configuration (AF), where electronic states with opposite spin are highly localized at the two GNR edges [36, 39, 40, 48, 49] and are responsible for the opening of a gap. It was shown that the magnetic instability energy of ZGNRs, i. e. the energy difference between the ferromagnetic and the non magnetic ground state, increases from $\sim 0.25 \mathrm{eV} /$ unit cell up to $\sim 0.37 \mathrm{eV} /$ unit cell when $N$ increases from 5 to 30 , and then stabilizes [40]. The energy difference between the ferromagnetic and the
AF ground state, however, are much smaller, with the AF state being more stable than the ferromagnetic by $4.0,1.8,0.4 \mathrm{meV} /$ unit cell when $N$ is $8,16,32$ [39]. Since $k_{B} T$ at room temperature corresponds to $\sim 25 \mathrm{meV}$, this indicates that ZGNRs at room temperature are stable in a magnetic state. As temperature decreases the antiferromagnetic state becomes favored over the ferromagnetic one. Thus DFT predicts both AGNRs and ZGNRs to always have a direct nonzero band gap at least at low temperatures [39, 40, 50]. In CNTs the gap scales inversely with diameter [76]. Similarly, in GNRs the gap scales inversely with the width [39, 40, 41, 51, 52], closing, as expected, for infinite graphene.

The electronic structure of GNRs was also studied using GW calculations [53, 54]. As for CNTs [77], the better description of the electron-electron interaction provided by GW increases the computed GNRs electronic gap. Furthermore, calculations based on the Bethe-Salpeter equation proved that, due to the presence of excitons, in GNRs the optical gap is significantly smaller than the single particle one [53, 54]. However, apart from the gap renormalization, all the main results obtained within DFT, including the AF ground-state of ZGNRs and the width dependence of the bandgap, are confirmed by the computationally more expensive GW [53, 54].

The presence of a gap inversely proportional to the ribbon's width was experimentally observed, for ribbons in the 10 to $500 \mathrm{~nm}$ range, by means of temperature dependent conductance $(\mathrm{G})$ measurements $[8,9,68]$. The gaps $\left(E_{g}\right)$ measured in these experiments are larger than those predicted by theory, and their width scales as $E_{g}=A(W$ $W *)^{-1}$, with $W$ being the ribbon's width and $A$ and $W^{*}$ fit constants [8]. In the same samples, the conductivity $G$ was found to scale as $G=B\left(W-W_{0}\right)$, with $B$ and $W_{0}$ fit constants. For $\mathrm{T}=1.6 \mathrm{~K}$, it was found that $W^{*} \sim W_{0}=16 \mathrm{~nm}[8]$. This suggests that structural disorder at the edges caused by etching, or inaccuracies in the width determination due to over-etching underneath the mask, can result in an "effective transport width" smaller than the ribbon's nominal width. Within this picture, $W_{0}$ and $W^{*}$ can be considered as the reduction of the effective width with respect to the nominal width [8]. Based on these results, some authors have also proposed that the size of the GNRs bandgap can be explained not only by confinement, but also by Coulomb blockade originated by the roughness at the edges of the ribbons [44]. This would also determine the value of $W^{*} \sim W_{0}$ [44]. Furthermore, it was also found that the minimum conductivity as a function of the gate changes with the environment or the GNR thermal history [9].

Finally, the electronic structure of mono-atomic step edges of graphite was also probed by scanning tunnelling microscopy (STM) and spectroscopy (STS). This showed a large electronic density of states (DOS) at the Fermi energy $\left(E_{F}\right)$ of zigzag steps, while a bangap was observed on armchair edges [42, 43, 45, 79]. These results seem to corroborate the simple tight-binding models predicting an accumulation of electronic states at $E_{F}$ in 
ZGNRs [36, 42, 43, 46], and to contradict the presence of the AF bandgap predicted by DFT and GW. However, it is important to recall that such experiments were performed on monoatomic steps of bulk graphite, which correspond to a semi-infinite ribbon. Since the onset of an antiferromagnetic state requires the presence of wavefunction with opposite spins localized on the two opposite edges of a ribbon, such state cannot be present in a semi-infinite system with just a single edge.

\section{B. Spin properties}

Ref. [50] showed, using DFT, that applying an electric field parallel to the ribbon's plane results in an opposite local gating of the spin states on the two edges of the ZGNR. The required critical field was estimated to be $3.0 \mathrm{~V} / W, W$ in $\AA[50]$. Indeed, the in-plane field lifts the spin degeneracy by reducing the band gap for one spin channel, while the other experiences a gap widening. This promotes the onset of half-metallicity [68, 80] or induces a spin-selective semiconducting behavior, where the band gap of one spin is bigger than the other. Which of these states is established depends on the intensity of the applied field [50, 55, 56] and on the ribbon's length $[56,57,58]$. These phenomena may have important applications for the fabrication of spin filtering devices[81].

Half-metals are materials in which the current can be completely spin polarized as a result of the coexistence of a metallic nature for electrons with one spin orientation and an insulating nature for electrons with the other spin [50, 68]. Some ferromagnetic metals were predicted to have this behavior [68] and were first observed experimentally in a manganese perovskite [80]. Similarly, there can be half semiconducting states in which the electron spins in the valence and conduction bands are oppositely polarized [67]. Since the development of efficient devices for spin injection, transport, manipulation and detection is a fundamental requirement for the incorporation of spin into existing semiconductor technology and to achieve spin based quantum computing and spintronics [81], substantial efforts were made in to find half-metallic materials [81, 82]. It is thus of great interest to identify such states in GNRs.

\section{Chemical modifications}

Chemical functionalization and substitutional doping have been investigated for many years in nanotubes 69, $70,71,72,83,84$, 85], with the aim of tailoring their properties for sensing, transport, chemical and optical applications or to incorporate them into polymers $[86,87,88,89,, 90]$. It is thus natural to do a similar investigation for GNRs.

Various theoretical studies of the influence of edge geometry and chemical modifications on the transport properties of ribbon junctions have been reported so a)
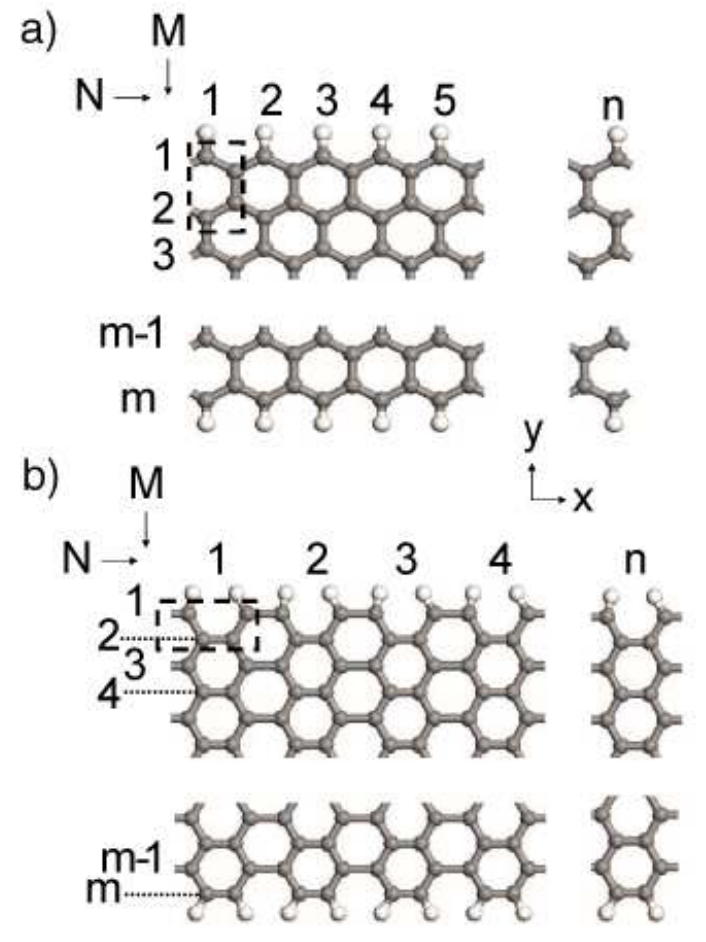

Figure 1: Scheme of $\mathrm{H}$ terminated (a) zigzag, and (b) armchair nanoribbons. Periodic boundary conditions are assumed in the x direction. $N$ and $M$ are the numbers of "colums" and "rows" of atoms used to label the $M \times N$ ribbon.

far [59, 60, 91, 92, 93, 94]. Furthermore, several works considered the reactivity of the ribbon edges and the effects of geometrical and chemical modifications on their electronic properties $[35,51,55,61,62,63,95,96,97,68$, 99, 100, 101, 102]. In particular, edge functionalization with oxygen-containing groups [55] and edge doping or edge imperfections [103] were shown to significantly lower the electric field required to induce half-metallicity in ZGNRs [55], to break the spin symmetry and to promote the possibility of net spin injection [103]. B substitutional doping [62, 63], either on the edge or in the bulk of GNRs, was reported to cause metal-semiconductor transitions in the ferromagnetic and non-magnetic ZGNRs states [62, 104]. The effect of B on the AF ground state has not yet been studied.

Also of interest is the effect of edge radical functionalization [99, 101]. Carbon atoms at the edges of ZGNRs offer superior chemical reactivity for the attachment of chemical groups than those on AGNRs edges, in the ribbon's bulk, or on CNTs edges [99, 105]. Indeed, as in the case of herringbone and bamboo-shaped CNTs [106, 107], the reactivity of GNRs is due to the presence of openended graphene sheets, which, in the case of ZGNRs, are particularly active thanks to the presence of electronic states highly localized at the edge carbon atoms [42, 99].

We note that transport and Raman measurements show that as-prepared graphene samples and devices reach the charge neutrality point for gate voltages dif- 
ferent from zero [23, 31, 73, 74, 78, 108]. Most times this corresponds to a shift of the Fermi level towards the valence band (indicative of p-doping) [23, 73, 74], but sometimes also towards the conduction band (equivalent to n-doping) [78]. In particular, refs. [73, 74] seem to indicate a bigger intrinsic p-doping for smaller ribbon size. Whether this behavior is a consequence of chemical doping due to edge functionalisation during lithography, or of a rearrangement in the electronic structure due to the presence of defect states away from the Fermi energy, or, as for large graphene samples, due to the presence of adsorbates [23, 108] needs to be further investigated.

\section{METHODOLOGY}

We perform spin polarized $a b$ initio calculations with the CASTEP plane wave DFT code [109] on hydrogen terminated ribbons. We use the Perdew-Burke-Ernzerhof gradient corrected functional [110] and ultrasoft pseudopotentials [111] with cut-off energies of $400 \mathrm{eV}$. Ribbons are built from the perfect graphite geometry with an initial C-C distance of $1.42 \AA$. Geometry optimization is performed for all the structures with the BroydenFletcher-Goldfarb-Shanno (BFGS) algorithm [112] until all the forces are smaller than $0.04 \mathrm{eV} / \AA$ and the stress in the periodic direction is less than $0.05 \mathrm{GPa}$.

A 4-atom unit cell is used to build our ZGNRs as shown in Fig. 11(a) and the same cell rotated $90^{\circ}$ is used to build the AGNRs of Fig. 1(b). The brillouin zones (BZ) of the GNR unit cells are sampled by Monkhorst-Pack [113] grids of the form $P \times 1 \times 1$ with $P$ such that the maximum spacing between $k$-points in the periodic direction $x$ (Fig. 11) is $<0.1 \AA^{-1}$. We find that, to simulate isolated ribbons, the in plane and perpendicular distances between ribbons in adjacent super-cells have to be larger than $5.5 \AA$ and $6.5 \AA$, respectively. The band structure is calculated with an eigenvalue tolerance of $0.001 \mathrm{meV}$.

We classify the ribbons with a $M \times N$ convention, where $M$ and $N$ are the number of rows and columns across the GNR width and length, respectively, as shown in Fig. 1. Throughout this work, we refer to edge functionalized ribbons with the $M \times N$ index of the clean GNR preceded by the chemical symbol of the functionalizing radical and followed by the character $Z$ or $A$ denoting the zigzag or armchair chirality. Thus, e.g., $\mathrm{NH}_{2}$ on the edge of a $2 \times 5$ zigzag ribbon is denoted as $\mathrm{NH}_{2}-2 \times 5 \mathrm{Z}$.

Atom substituted ribbons are labeled by the symbol of the substitutional atom followed by the word bulk or edge, to denote bulk or edge substitution, and the $M \times N$ index of the ribbon. For example $\mathrm{N}$ bulk substitution in the $6 \times 5$ AGNR is denoted as Nbulk- $6 \times 5 \mathrm{~A}$.

\section{RESULTS}

\section{A. Edge functionalisation: single edge}

Due to the presence of edge states in ZGNRs and their absence in AGNRs, the effects of single-edge radical functionalization strongly differ. Indeed, as shown in the following and in Figs. 22(a-f), 5(a), functionalization of ZGNRs lifts the spin degeneracy and in some cases also promotes a semiconductor-metal transition, while in AGNR the largest effect is the presence of impurity levels deep in the valence band that hardly modify the gap.

We start by investigating five radical groups on the $6 \times 2$ ZGNR. The top insets in Fig. 2(a-f) schematize functionalisation with (a) $\mathrm{NH}_{2}$, (b) $\mathrm{OH}$, (c) $\mathrm{COOH}$, (d,e) $\mathrm{NO}_{2}$ and (f) $\mathrm{O}$. In general, geometry optimization shows that edge functionalization does not alter significantly the GNRs structure. For these radicals, we find the following optimized geometries: the $\mathrm{NH}_{2}$ sits $1.386 \AA$ away from the edge carbon, the $\mathrm{H}-\mathrm{N}$ distances are $1.021 \AA$ and the $\mathrm{H}-\mathrm{N}-\mathrm{H}$ plane is tilted $\sim 20^{\circ}$ with respect to the plane of the ribbon. The $\mathrm{OH}$ remains on the graphene plane with $\mathrm{C}-\mathrm{O}$ and $\mathrm{O}-\mathrm{H}$ distances of $1.367 \AA$ and 0.977 $\AA$ respectively and a $\mathrm{C}-\mathrm{O}-\mathrm{H}$ angle of $\sim 109^{\circ}$. The plane defined by the $\mathrm{O}-\mathrm{C}-\mathrm{O}$ of the $\mathrm{COOH}$ has an angle of $\sim 53^{\circ}$ with respect to the plane of the ribbon and the $\mathrm{C}-\mathrm{C}$, $\mathrm{C}-\mathrm{O} 1, \mathrm{C}-\mathrm{O} 2$ and $\mathrm{O} 2-\mathrm{H}$ distances are $1.487 \AA, 1.229 \AA$, $1.365 \AA$ and $0.985 \AA$ respectively. For $\mathrm{NO}_{2}$ we find two equilibrium geometries: one energetically stable $(\mathrm{S})$ and one metastable (MS), separated by $0.407 \mathrm{eV}$. The $\mathrm{S}-\mathrm{NO}_{2}$ has $\mathrm{C}-\mathrm{N}$ and N-O distances of $1.466 \AA$ and $1.257 \AA$, and the $\mathrm{O}-\mathrm{N}-\mathrm{O}$ plane is tilted $\sim 60^{\circ}$ with respect to the cross section of the ribbon. The $\mathrm{MS}-\mathrm{NO}_{2}$ sits in a configuration similar to the $\mathrm{NH}_{2}$, with $\mathrm{C}-\mathrm{N}$ and $\mathrm{N}-\mathrm{O}$ distances of $1.466 \AA$ and $1.262 \AA$. Finally, for the functionalization with a single $O$, this sits in the ribbon's plane in front of the edge $\mathrm{C}$ with $\mathrm{C}$-O distance $1.245 \AA$.

Functionalization strongly affects the electronic structure of ZGNRs. This is shown in Fig. 2(a-f), where the density of states (DOS) of a set of functionalized ribbons is compared with the corresponding DOS of the clean ribbon. Single-side radical edge functionalization of a ZGNR (Figs. 24) has an effect on the ribbon's spin density and band structure similar to that of an electric field parallel to the ribbon's plane 50, 55, 56, 57]. Thus, the presence of functional groups, while preserving an AF ground state [39, 51], lifts the spin degeneracy of the clean ribbons [101]. For the radical density we use, this results in the onset of spin-selective semiconductivity in $\mathrm{NH}_{2}, \mathrm{OH}, \mathrm{COOH}$ and $\mathrm{S} \mathrm{NO}_{2}$ functionalizations, and of a semiconductor-metal transition for $\mathrm{O}$ and $\mathrm{MS} \mathrm{NO}_{2}$.

The lifted spin degeneracy implies that in all cases the band gaps of the two spin channels, which we call $\alpha$-spin and $\beta$-spin, are different (Fig. 2, $4 \alpha$-red/gray solid line and $\beta$-blue/black solid line). The change of gap with respect to the original $\mathrm{H}$ terminated GNR fingerprints the radical used to functionalize the ribbon. This is shown in Fig. 2(a-f), which plots the DOS for each of the five rad- 


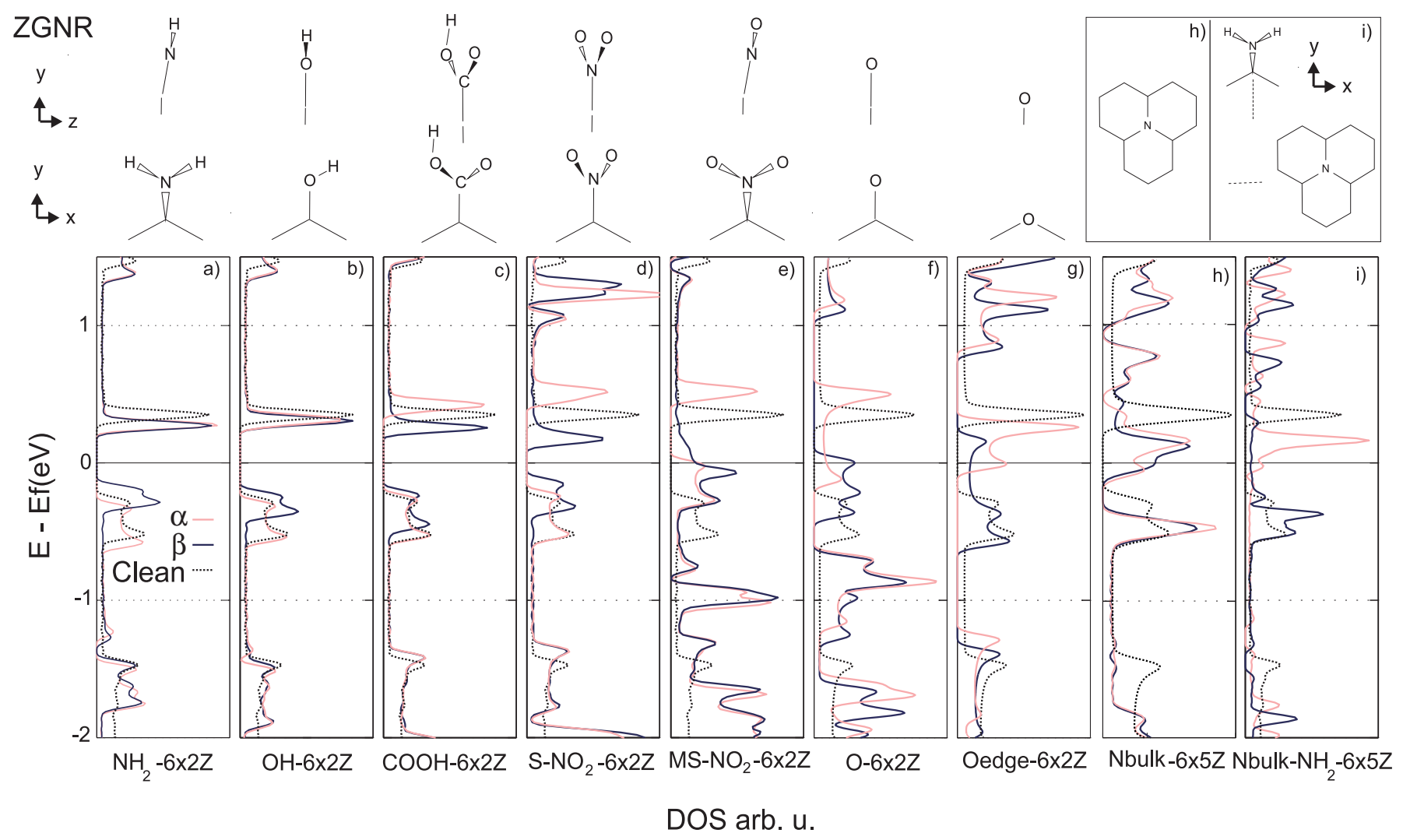

Figure 2: (Color online) Spin density of states of $\alpha$ (red/gray solid line) and $\beta$ (blue/black solid line) for functionalized ZGNRs in comparison with clean ribbon (black dotted line). (a-f) $6 \times 2 \mathrm{Z}$ (i. e. $M=6, N=2 \mathrm{ZGNR}$ ) functionalized with (a) $\mathrm{NH}_{2}$, (b) $\mathrm{OH}$, (c) $\mathrm{COOH}$, (d) Stable- $\mathrm{NO}_{2}$, (e) Meta Stable- $\mathrm{NO}_{2}$ and (f) O radicals in one of the ribbon edge producing breakage of the spin degeneracy(a-f) spin-selective band gap(a-d) or semiconductor-metal transition (e,f). (g) Oxygen edge substitutional atom favoring a semiconductor-metal transition. (h) $6 \times 5$ zigzag ribbon with a nitrogen bulk substitution in the center of the ribbon. (i) Nitrogen bulk substituted ribbon shown in (h) with an extra single $\mathrm{NH}_{2}$ at the edge of the opposite carbon sublattice, with the maximum distance between $\mathrm{N}$ and $\mathrm{NH}_{2}$ in the periodic direction presenting semiconductor-metal transition

icals. A pristine $6 \times 2 \mathrm{Z}$ ribbon has a $0.58 \mathrm{eV}$ direct band gap at $k=0.5 \pi / c, c$ being the size of the ribbon's unit cell along its periodic direction. The different functionalizations change the band gap as follows. In presence of $\mathrm{NH}_{2}$ the gap remains direct and at $k=0.5 \pi / c$ for both spins, but shrinks to $0.42 \mathrm{eV}$ and $0.39 \mathrm{eV}$ for the $\alpha$ and $\beta$ spin channels, respectively. A similar effect is obtained by means of $\mathrm{OH}$ functionalisation, with final gaps of 0.57 $\mathrm{eV}$ and $0.49 \mathrm{eV}$. In the case of $\mathrm{COOH}$, the direct gap at $k$ $=0.5 \pi / c$ increases up to $0.62 \mathrm{eV}$, but an indirect one of $0.45 \mathrm{eV}$, with extremes at $k=0$ in the conduction band and $k=0.5 \pi / c$ in the valence band, appears. Similarly, $\mathrm{S}-\mathrm{NO}_{2}$ gives a direct gap of $0.66 \mathrm{eV}$ and a smaller indirect gap of $0.28 \mathrm{eV}$ with the extremes located at the same position as the indirect gap due to $\mathrm{COOH}$. $\mathrm{MS}_{-} \mathrm{NO}_{2}$ and $\mathrm{O}$ have several extra levels in the valence band and a zero gap for both spin channels. This makes them metallic.

The semiconductor-metal transition we predict for $\mathrm{O}$ functionalized ZGNRs is an intriguing. Ribbons are often produced by oxygen plasma etching [78], suggesting that the same technique used to cut ribbons, could also influence their electronic properties. We thus perform a calculation for a lower edge functionalization density. We find that for a $6 \times 6 \mathrm{Z}$ ribbon the semiconductor-metal transition does not happen, but we have a gap of $0.82 \mathrm{eV}$ and $0.41 \mathrm{eV}$ for the $\alpha$ and $\beta$ spins, respectively $(+0.40 \%$ and $-30 \%$ with respect to pristine ribbon). This implies that the semiconductor/metallic behavior of the ribbon could be controlled by the degree of oxidation of a single zigzag edge, provided this does not revert to $\mathrm{OH}$, which, as discussed before, would not significantly affect the gap.

We note that, while $\mathrm{NO}_{2}$ and $\mathrm{NH}_{2}$ on graphene act as strong acceptor and donors [108, 114], the same molecules at the edges of the ZGNRs modify the electronic structure in the gap vicinity, but do not act as dopants.

Fig. [3 shows the effect of functionalization on the spin density and band structure of both single- and doubleside functionalized ZGNRs. Black dotted lines represent the bands of the $2 \times 1(4 \times 1)$ clean ZGNRs folded into the $2 \times 2(4 \times 8)$ reciprocal unit cell. The band structure of the clean ribbons is spin degenerated [39, 51], and the spin density is distributed in an $\mathrm{AF}$ arrangement from the edges to the center, as in Refs. 39, 51]. Fig 3(a,d) indicates that single-edge $\mathrm{NH}_{2}$ functionalization introduces 

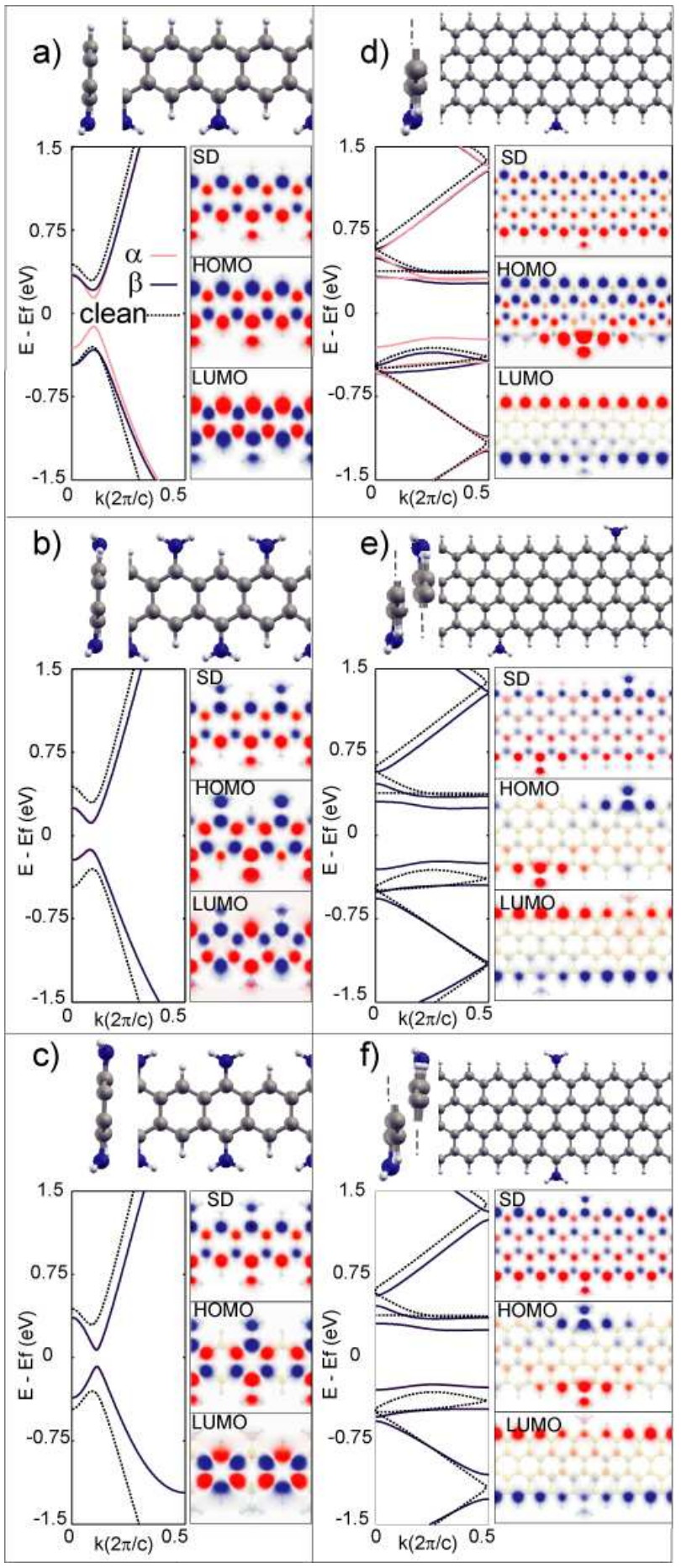

Figure 3: (Color online) Band structure of the $\mathrm{NH}_{2} 2 \times 2 \mathrm{Z}$ (ac) and $4 \times 8 \mathrm{Z}$ (d-f) single-side (a,d) and double-side (b,c,e,f) functionalization. Single side functionalization (a,d) breaks spin degeneracy, whereas this is nearly recovered for doublesided antisymmetric (b,e) and symmetric (c,f) functionalization. $\alpha(\beta)$ spins are represented by red/gray(blue/black) solid lines. The folded band structures of the pristine ZGNRs are plotted with black dotted lines. On the right of each BS are the corresponding spin density maps ( $\beta$-blue and $\alpha$-red) of the total spin density (SD), spin density of the HOMO and LUMO bands. The image shows the relaxed structure in $x y$ projection and a close up of the edges in the $y z$ projection. extra charges that alter the spin density. Due to the zigzag geometry, $\mathrm{C}$ atoms sitting on the outmost position of opposite edges always belong to different sublattices. As a consequence, single edge functionalization affects prevalently the spin channel localized on the same edge where the functional group is attached. This is shown in Fig. 3(a,d). Indeed, after functionalization, the two sublattices are visible in the total spin density (SD) maps with the characteristic AF spin arrangement. However, $\mathrm{NH}_{2}$ alters the SD, attracting $\alpha$ spin regions in its vicinity, while the $\beta$ spin density is substantially unchanged.

The perturbation of the SD is reflected in the lifting of the spin degeneracy illustrated in the DOS plot of Fig. 2, and in the band structure of Fig. 3) (a,d). As the highest occupied molecular orbital (HOMO) and lowest unoccupied molecular orbital (LUMO) are highly localized on the edge of the ZGNRs [35, 39], the influence of edge functionalization is predominantly on these orbitals. This is shown by the maps of the total spin density and of the HOMO and LUMO spin denisity in Fig. 3(a,d). For the valence band orbitals of the $2 \times 2$ and $4 \times 8$ ribbons, the $\alpha$ spin (red/gray) hosting the $\mathrm{NH}_{2}$ presents a stronger modification than the $\beta$ spin (blue/black) orbital in the opposite edge. Looking to the corresponding band, the $\alpha$ HOMO is more modified than the $\beta$ HOMO with respect to the original HOMO (black dotted line). The breakage of the spin degeneracy at the top of the HOMO band is $0.21 \mathrm{eV}$ and $0.13 \mathrm{eV}$ for the $\mathrm{NH}_{2}-2 \times 2 \mathrm{Z}$ and $\mathrm{NH}_{2}-4 \times 8 \mathrm{Z}$ ribbons respectively (note the color matching between spin density maps and spin bands in all Fig. 31). We expect similar effects for any $N, M$ combination and for any radical attached to only one ribbon edge.

We finally consider edge radical functionalization on AGNRs. AGNRs do not have edge states near the band gap, so the effect is expected to be weaker than in ZGNRs. Fig. 5(a) shows $\mathrm{NH}_{2}$ functionalization on the edge of the $12 \times 3$ AGNR. Here an impurity level is found $\sim 1.5 \mathrm{eV}$ below the edge of the valence band, the gap increases by $+0.02 \mathrm{eV}(\sim 4 \%)$, with no lifting of the spin degeneracy. Thus, for AGNR, $\mathrm{NH}_{2}$ edge functionalization does not significantly affect the electronic properties, since the impurity level is located deep in the valence band and no other major change happens to the DOS. We expect that functionalization with other chemical groups will give similar results. Nevertheless, finding a chemical group able to introduce impurity levels in the gap will be of great interest for electronic applications, so further investigations are desirable.

\section{B. Edge functionalisation: double edge}

The effects of double edge functionalization are shown in Fig. 3(b,c,e,f). Interestingly, when another radical of the same species is located in the opposite edge of the ribbon, the spin degeneracy is nearly restored . Fig. 3(b,e) show the $4 \times 8 \mathrm{Z}$ and $2 \times 2 \mathrm{Z}$ ribbons with two $\mathrm{NH}_{2} \mathrm{impu}-$ rities on opposites edges in an asymmetric arrangement. 
a)

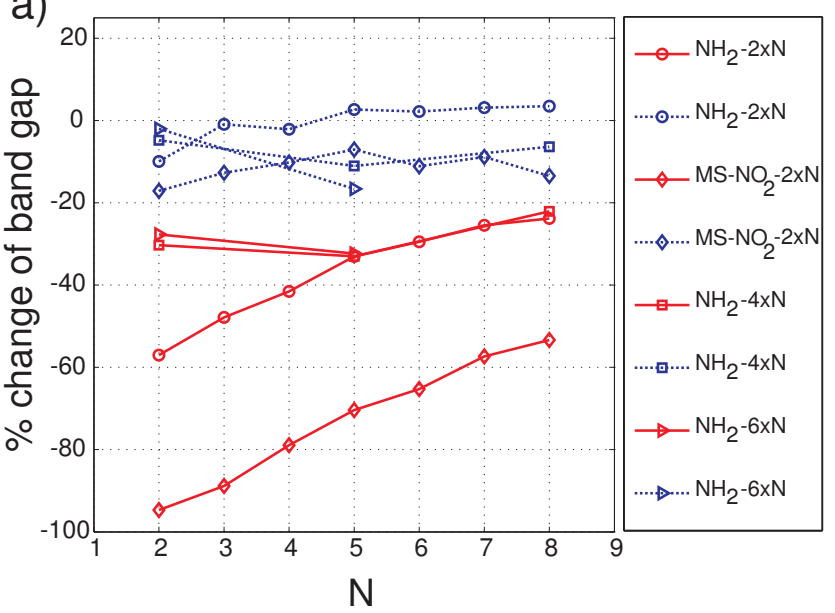

b)

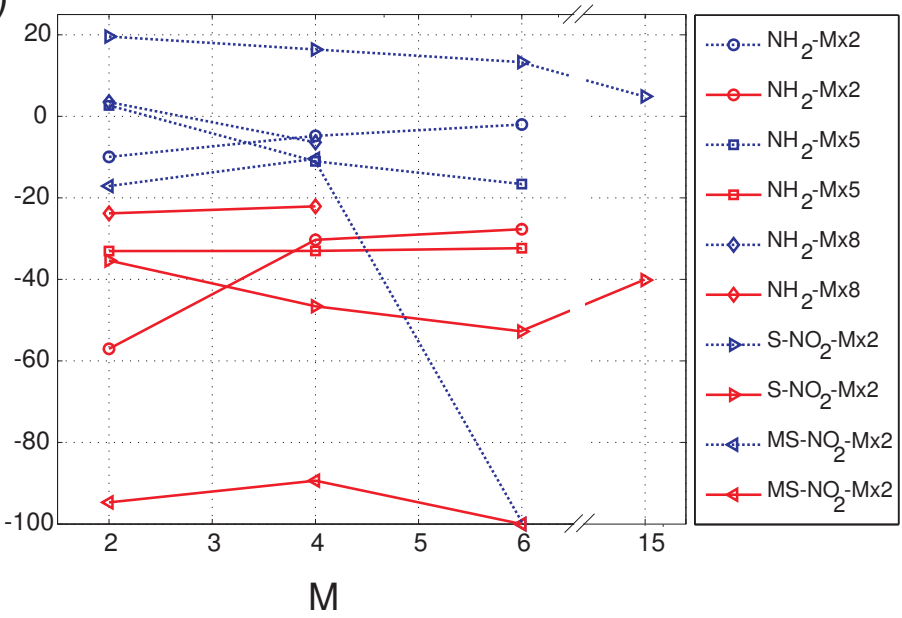

Figure 4: (Color online)Change of spin band gap ( $\beta$ channel-blue dotted line, $\alpha$ channel-red solid line) as a function of (a) edge density $-N$ - and (b) width of ribbon $-M$ - for different set of edge functionalized ZGNRs.

The breakage of the degeneracy between $\alpha$ and $\beta$ HOMO is only $1 \mathrm{meV}$ and $0.4 \mathrm{meV}$ for the double side asymmetric functionalized $2 \times 2 \mathrm{Z}$ and $4 \times 8 \mathrm{Z}$ ribbons, respectively. Fig. 3(c,f) show the same ribbons with two $\mathrm{NH}_{2}$ impurities sitting on opposite edges in a symmetric arrangement. In this case the breakage of the spin degeneracy between $\alpha$ and $\beta$ HOMO bands is $11 \mathrm{meV}$ and $3.6 \mathrm{meV}$ for the $2 \times 2 \mathrm{Z}$ and $4 \times 8 \mathrm{Z}$ ribbons respectively.

The differences between the symmetric and antisymmetric geometries indicate the different stability of these configurations. Indeed, the total energy of the antisymmetric ribbon is lower than that of the symmetric ribbon by $42 \mathrm{meV}$ and $34 \mathrm{meV}$ for the $2 \times 2 \mathrm{Z}$ and $4 \times 8 \mathrm{Z}$ ribbons.

To restore spin degeneracy in 2 -sided functionalized ribbons, it is necessary to have the same impurity density on both edges, and the maximum separation between consecutive radicals of opposite edges has to be achieved. Finally, it is important to stress that any geometry distortion or irregularity will result in a stronger magnetization of one of the edges, thus lifting the spin degeneracy.

\section{Edge functionalisation: density of radicals dependence}

We now consider the effects of the spacing between radicals, which is proportional to the index $N$, on the electronic structure of one-sided functionalized ZGNRs. Fig. 4(a) plots, for a set of $\mathrm{NH}_{2}$ and $\mathrm{NO}_{2}$ functionalized ribbons, the change in the band gap for each spin as a function of $N$. A high level of $\mathrm{NH}_{2}$ functionalization of the $M=2$ ribbon can shrink the band gap of the $\alpha$ spin up to $\sim 60 \%$, while leaving the $\beta$ gap almost unchanged.

A similar behavior is observed for the MS- $\mathrm{NO}_{2}-2 \times \mathrm{NZ}$ ribbon. In this case, increasing the functionalization densities narrows the $\alpha$ gap up to almost its complete closure, while the $\beta$ gap has a maximum change of $\sim-20 \%$, almost independent of doping. These results, obtained for very thin ribbons $(M=2)$, seem to suggest a correlation between the breakage of the spin degeneracy and the edge-functionalization density, the former slowly decreasing for increasing $N$. However, in wider ribbons (e.g. $M=4,6$ ), the band gap shows a much weaker dependence on $N$. In particular, for the $\mathrm{NH}_{2}-4 \times N$ ribbon the changes in the $\alpha$ and $\beta$ gaps oscillate between $20-35 \%$ and $3-18 \%$ respectively when $N$ spans between 2 and 8. Almost identical gap variations are observed for $\mathrm{NH}_{2}-6 \times N Z$ for $N$ between 2 and 5 .

\section{Edge functionalisation: width dependence}

Fig. 4(b) plots the variation of the $\alpha$ and $\beta$ bandgap, relative to the size of the bangap in the clean ribbon, in single-side functionalized ribbons as a function of $M$. Fig. [4] suggest that the modulation of the gap due to functionalization depends very on $M$, although some strong fluctuations may still occur for the thinnest ribbons (i.e. $M=2$ ). The points for $M=15$ in Fig. 4(b) confirm this behavior, showing that the variation of the $\alpha$-spin gap due to functionalization in the $\mathrm{S}^{-\mathrm{NO}_{2}}-M \times 2 \mathrm{Z}$ ribbons is $-32 \%$ to $-55 \%$ for $M$ changing from 2 to 6 . A further increase of $M$ from 6 to 15 (ribbon width increasing from $1.3 \mathrm{~nm}$ to $3.8 \mathrm{~nm}$ ) leaves the functionalization contribution almost unchanged for both spin channels. Fig. 4 thus confirms that edge functionalization always promotes lifting of the spin degeneracy, and indicates that such effect decays slowly as a function of $M$.

A remarkable exception are the $\mathrm{MS} \mathrm{NO}_{2}$-ZGNRs. The MS- $\mathrm{NO}_{2}-2 \times 2 \mathrm{Z}$ and $\mathrm{MS}-\mathrm{NO}_{2}-4 \times 2 \mathrm{Z}$ are half semiconducting, and the $\alpha$ and $\beta$ gaps are respectively reduced to $\sim 90 \% \sim 15 \%$ of the original gap, while the $\mathrm{MS}-\mathrm{NO}_{2}-$ $6 \times 2 \mathrm{Z}$ ribbon is metallic for both spin channels. 


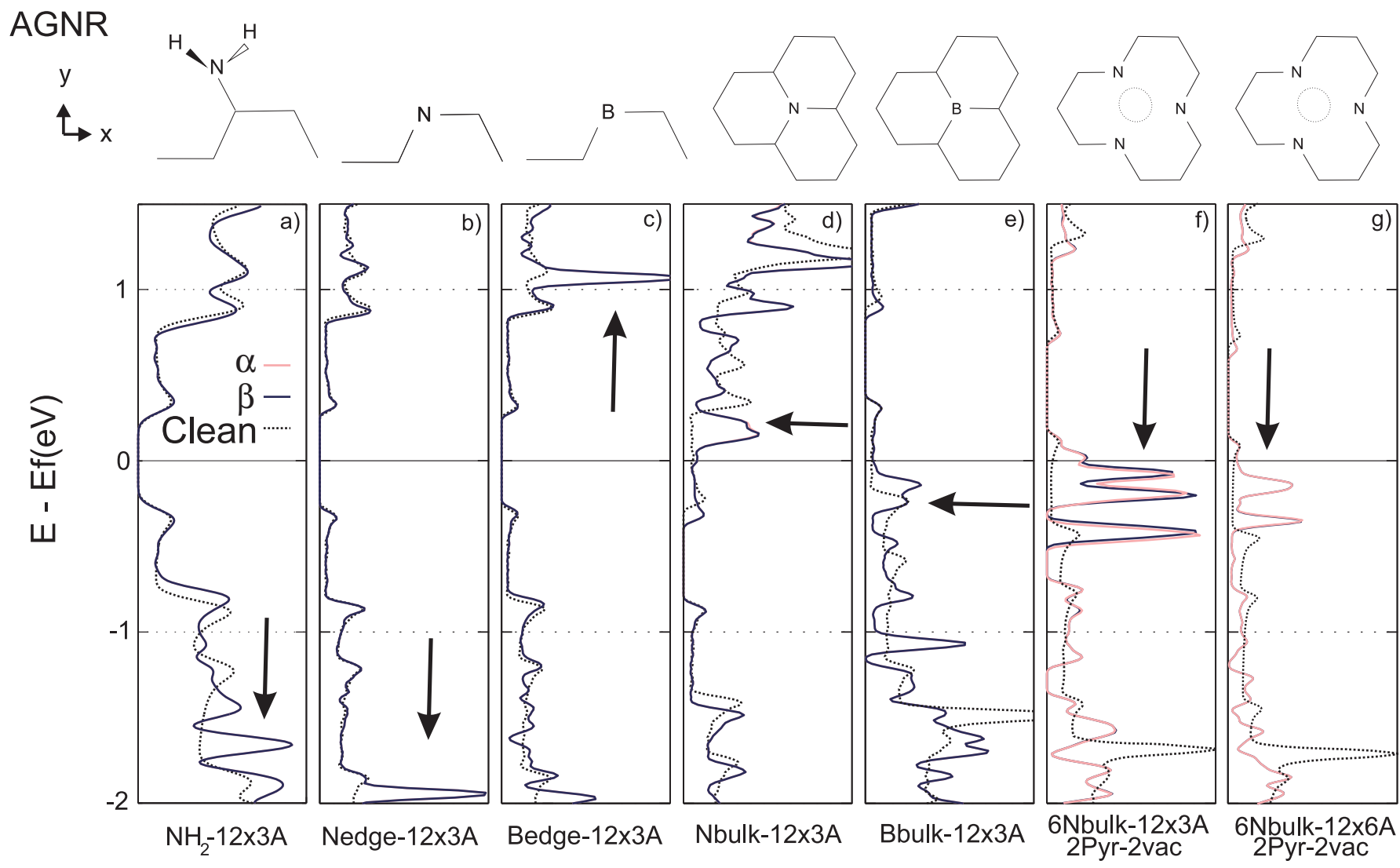

DOS arb. u.

Figure 5: (Color online) Spin density of states of $\alpha$ (red/gray solid line) and $\beta$ (blue/black solid line) for functionalized AGNRs in comparison with clean ribbon (black dotted line). (a) $12 \times 3$ AGNR functionalized in one edge with $\mathrm{NH}_{2}$. (b) $\mathrm{N}$, (c) $\mathrm{B}$ edge substitutional atoms on the $12 \times 3$ AGNR. N and B bulk substitution for the $12 \times 3$ AGNR is shown in (d) and (e), where a semiconductor-metal transition occurs. (f,g) two Pyridine-like substitutional doping in the bulk of 2x3a and 12x6a presenting metal behavior. Arrows indicate impurity levels due to substitutional atoms. The DOS of the clean ribbon is shifted for the bulk $\mathrm{N}$ and $\mathrm{B}$ substitution such that the edge of the valence band of clean and doped ribbons coincide

\section{E. Atomic substitution}

In semiconducting CNTs, substitutional B [69, 70] and $\mathrm{N}$ [70, 71] act as acceptors or donors, inserting levels in the gap or causing a semiconductor-metal transition. B substitution on the edge of meta-stable ferromagnetic ZGNRs was shown to induce metal-semiconductor transitions 62]. It is thus interesting to study in more detail the effects of atomic substitutions in GNRs.

We start by considering $\mathrm{O}$ substitution on the edge of ZGNRs. By using a $6 \times 2 \mathrm{Z}$ ribbon, we find that in the optimized geometry the two $\mathrm{C}-\mathrm{O}$ distances are $1.41 \AA$. The resulting DOS is reported in Fig. 2(g), showing that the appearance of new states in the conduction band promotes the onset of a semiconductor-metal transition.

To investigate the role of the functionalization density, we repeat the same calculation for a $6 \times 6 \mathrm{Z}$ ribbon. In this case, no semiconductor-metal transition is observed. Instead, the spin degeneracy is lifted, and the band gap for the two spin channels are $0.449 \mathrm{eV}$ and $0.399 \mathrm{eV}$, which represent a change of $-22 \%$ and $-31 \%$ with respect to the pristine ribbon. Overall, the effects of $\mathrm{O}$ functionalization (Fig. 2(f)) and edge substitution (Fig. 2(g)) differ only for the the position of the extra levels. Since in the center of the ribbon the spin density is much lower than the edges, the effect of bulk substitutions on the breakage of the spin degeneracy is expected to be weaker than the corresponding edge substitution or edge functionalization. Fig. 2(h) shows the effect of $\mathrm{N}$ substitution in the center of a $6 \times 5 \mathrm{Z}$ ribbon, which corresponds 1.7 at\%, i.e. $1 \mathrm{~N}$ atom in a lattice of $59 \mathrm{C}$ atoms. This gives a semiconductor-metal transition, with a weak spin degeneracy lifting. However, as shown in Fig. 2(i), the introduction on the edge of the same ribbon of a $\mathrm{NH}_{2}$ radical leads to a much more pronounced difference between the two spin channels DOS. In this case, the N substitutional atom is placed in the opposite carbon sub-lattice with respect to $\mathrm{NH}_{2}$, and the separation between $\mathrm{N}$ and the $\mathrm{NH}_{2}$ radical is maximized. This arrangement is chosen with the aim to alter the AF state of both sub-lattices.

We now consider low concentration atomic substitution 
on the edge and bulk of AGNRs. For edge substitution we use the $12 \times 3$ AGNR ( 1 substitutional atom in $71 \mathrm{C}$ atoms, 1.4 at\%). In this case $\mathrm{N}$ impurity states appear $\sim 1.6 \mathrm{eV}$ below the valence band (Fig. 5(b)) and the B states $\sim 0.8 \mathrm{eV}$ above the conduction band (Fig. 5)(c)). The impurities modify the gap by $+8.7 \mathrm{meV}(+1.5 \%)$ for $\mathrm{N}$ and $-32 \mathrm{meV}(-5 \%)$ for $\mathrm{B}$. In the relaxed structure the two N-C nearest neighbor distances are $1.33 \AA$ and 1.36 $\AA$ and the B-C bonds are $1.40 \AA$ and $1.43 \AA$.

Bulk substitution is then investigated in the $12 \times 3 \mathrm{AG}$ NRs. Here we observe that both $\mathrm{N}$ and $\mathrm{B}$ give impurity levels localized around the substituted atoms. This shifts the Fermi energy $\sim 0.5 \mathrm{eV}$ inside the former conduction and valence band, resulting in a semiconductor-metal transition. As indicated by the arrows in Fig. 5(d,e), the impurity bands are localized at $\sim 0.15 \mathrm{eV}$ from the Fermi energy. In the relaxed structure the N-C and B-C distances are $1.41 \AA$ and $1.49 \AA$, respectively.

Unlike CNTs [69, 70, 71], substitutional $\mathrm{B}$ and $\mathrm{N}$ in AGNRs do not give electronic states in the band gap, even if semiconductor-metal transitions can still be observed. Further investigations with different substitutional atoms or geometries are needed to determine if and how conventional doping of GNRs can be achieved.

We finally consider the effect of pyridine-like doping. This consists in the substitution of 4 carbon atoms by 3 nitrogens and a vacancy, as schematically shown in the diagram of Fig. $5(f, g)$. In CNTs, high density pyridine-like doping was found to introduce donor-like states above the Fermi energy and to produce semiconductor-metallic transitions [72]. We start by introducing 2 pyridinelike impurities in the $12 \times 3$ AGNR, corresponding to 9.4 at $\% \mathrm{~N}$. The vacancies are arbitrarily positioned near the center of the ribbon at 1 row and 2 columns of atoms away from each other. This gives 4 localized levels in the valence band near the Fermi energy, which induce a Fermi energy shift and cause a semiconductor-metal transition. We also consider two pyridine-like impurities in the $12 \times 6 \mathrm{~A}$ ribbon, corresponding to 4.4 at $\% \mathrm{~N}$. These are positioned in the nearest possible sites to the center of the ribbon at a inter-vacancy distance in the periodic direction of 5 columns of carbon atoms. The arrow in Fig. 5(g) shows the impurity levels in the valence band of the ribbon due to the pyridine-like doping. These levels, as in the case of higher impurity density, shift the Fermi energy and cause a semiconductor-metal transition.

\section{DISCUSSION}

The fabrication of devices with a new material requires the understanding all the factors that influence its electronic and spin properties. The geometry and the presence of various form of chemical modifications are crucial for GNRs. The geometry is defined by length, width, and edges chirality, whereas chemical modifications can be caused by different edge terminations, and by substitutional atoms in the ribbons' body.
In this paper, we examined how all these factors affect the electronic and spin properties of infinite ribbons. Our main result is that a strong spin polarization is maintained in all the simulated zigzag ribbons. In particular, our calculations show that single edge functionalization leads to half-semiconductors with different band gap for each spin and can also result in a spin-polarized halfsemiconductor or in a semiconductor-metal transition. This implies the possibility of using single edge functionalized ribbons to realize spin filtering devices, even though single-edge functionalisation may be experimentally very challenging. On the other hand, the weak dependence of spin degeneracy lifting and half-metallicity on width is encouraging for potential applications, since wider ribbons are easier to make.

We also observe that high concentrations of oxygens on the ribbons edges are likely to produce metallic ZGNRs, while for lower oxygen concentrations, ZGNRs are expected to be half semiconducting. Thus, oxygen is one of the most effective elements to promote the closure of the electronic gap for both spin channels. Since ribbons can be cut from bulk graphene by means of an oxygen plasma [8, 78], this could affect their final electronic properties. However, it is likely that $\mathrm{OH}$ may also result from this treatment. But $\mathrm{OH}$ does not close the gap. Given the large gaps so far reported [8, 78], it is thus unlikely that these GNRs are just $\mathrm{O}$ terminated.

It is known that the electronic structure of AGNRs does not present spin-polarization effects [35, 39] and that their gap scales inversely with the width [35, 39]. Our calculations indicate that the gap in AGNRs is extremely stable with respect to the edges chemical termination. This is due to the absence of edge states, which is, on the contrary, the main characteristic of zigzag ribbons. Armchair ribbons seem thus ideal for devices. However, our calculations indicate that the presence of $\mathrm{B}$ and $\mathrm{N}$ does not give defect states that pin the Fermi energy in the gap, showing that the behavior of AGNRs with respect to atomic substitutions is markedly different from that of CNTs. Indeed, even if it is well established that replacing $\mathrm{C}$ atoms with $\mathrm{B}(\mathrm{N})$ in semiconducting CNTs results in electronic states that pin the Fermi energy close to the conduction (valence) band, producing a $n(p)$-doping [69, 70, 71, 72, 83, 84], our simulations show that in AGNRs the same substitutions lead to completely different effects. If the substitution occurs on the ribbon edges, the impurity levels are always very far from the Fermi energy, and the electronic properties of the ribbon are substantially unchanged. If it occurs in the center, the defect states appear close to the bottom (top) of the conduction (valence) band of the pristine ribbon, but the Fermi energy is shifted in the conduction (valence) band, triggering a semiconductor-metal transition, rather than doping, unlike in CNTs and bulk semiconductors.

The physical reason for the different effects of chemical doping in GNRs and CNTs is likely to derive from the different boundary conditions to which the electronic wave-functions have to obey in the two materials. How- 
ever, further investigations are needed to fully address this point. The reason why edge substitutional atoms do not result in a shift of the Fermi energy is related to the relative position of defect states and the bandgap of the pristine ribbon. Indeed, $\mathrm{N}$ edge atoms are attached to the ribbon with very localized chemical bonds in an $s p^{3}$ configuration. This promotes the formation of a deep state in the valence band, which is occupied by the extra electron carried by the $\mathrm{N}$. On the contrary, $\mathrm{N}$ in the bulk of the ribbon is in an $s p^{2}$ configuration. This gives delocalized states in the conduction band. Since no new states are formed in the valence band, the extra electrons coming from $\mathrm{N}$ have to be allocated in what was the conduction band of the pristine ribbon, with a consequent shift of the Fermi energy. A perfectly specular consideration can be done for B doping.

Finally, we stress that here we focussed only on chemical modification of the ribbons, without taking into account additional effects, such as chemisorption or physisorption of molecules on the ribbons'surfaces. Since adsorbates can easily shift the fermi level in large graphene samples [108, 114, 115, 116, 117], the same could also happen for ribbons. This deserves further investigation, and may help understand some experimental observations, such as the tendency of small ribbons to appear positively charged [23, 73, 74] or the dependence of their minimum conductivity on the environment [9].

\section{CONCLUSIONS}

We studied atomic substitution and edge functionalization in graphene ribbons. The distinctive anti- ferromagnetic phase of the spin on the edges of zigzag nanoribbons is altered by any single edge functionalization, producing half-semiconducting structures. An appropriate functionalization with the correct radical could produce half-metallicity, since different radicals alter the anti-ferromagnetic arrangement in different ways. Oxygen edge substitution and functionalization at high density in zigzag nanoribbons produce semiconductor-metal transitions. Double edge functionalization of zigzag ribbons can reduce the semiconducting gap, with an antisymmetric arrangement of the radicals energetically favored. Nitrogen bulk substitution at 1.7 at\% in zigzag ribbons also makes them metallic. The gap of armchair ribbons is robust against edge functionalization, and N,B edge substitutions. However N, B and pyridine-like bulk substitution causes semiconductor-metal transitions.

\section{ACKNOWLEDGMENTS}

F.C.S. acknowledges funding from CONACYT Mexico. S.P. acknowledges funding from The Maudslay Society and Pembroke College, and A.C.F. from The Royal Society and The Leverhulme Trust.
[1] K. S. Novoselov, A. K. Geim, S. V. Morozov, D. Jiang, M. I. Katsnelson, I. V. Grigorieva, S. V. Dubonos, and A. A. Firsov; Nature (London), 438, 197 (2005).

[2] K. S. Novoselov, Z. Jiang, Y. Zhang, S. V. Morozov, H. L. Stormer, U. Zeitler, J. C. Maan, G. S. Boebinger, P. Kim, and A. K. Geim; Science, 315, 1379 (2007).

[3] K. S. Novoselov, A. K. Geim, S. V. Morozov, D. Jiang, Y. Zhang, S. V. Dubonos, I. V. Grigorieva, A. A. Firsov; Science, 306, 666 (2004).

[4] A. K. Geim, K. S. Novoselov; Nature Materials, 6, 183 (2007).

[5] A. H. Castro Neto, F. Guinea, N. M. R. Peres, K. S. Novoselov, A. K. Geim; arXiv:0709.1163v1.

[6] Y. Zhang, Y.W. Tan, H. L. Stormer, and P. Kim; Nature (London), 438, 201 (2005).

[7] S. V. Morozov, K. S. Novoselov, M. I. Katsnelson, F. Schedin, D. Elias, J. A. Jaszczak, A. K. Geim; arXiv:0710.5304.

[8] M. Y. Han, B. Özylmaz, Y. Zhang, P. Kim; Phys. Rev. Lett., 98, 206805 (2007).

[9] Z. Chen, Y.M. Lin, M. Rooks, P. Avouris; cond-mat/0701599

[10] Y. Zhang, J. P. Small, W. V. Pontius, P. Kim; Appl.
Phys. Lett., 86, 073104 (2005).

[11] M. C. Lemme, T. J. Echtermeyer, M. Baus, and H. Kurz; IEEE El. Dev. Lett., 28, 4 (2007).

[12] T. A. Land, T. Michely, R. J. Behm, J. C. Hemminger, G. Comsa; Surf. Sci., 264, 261 (1992).

[13] A. Nagashima, K. Nuka, H. Itoh, T. Ichinokawa, C. Oshima, S. Otani; Surf. Sci., 291, 93 (1993).

[14] S. Entani, S. Ikeda, M. Kiguchi, K. Saiki, G. Yoshikawa, I. Nakai, H. Kondoh, T. Ohta; Appl. Phys. Lett., 88, 153126 (2006).

[15] C. Berger, Z. Song, T. Li, X. Li, A. Y. Ogbazghi, R. Feng, Z. Dai, A. N. Marchenkov, E. H. Conrad, P. N. First, W. A. de Heer; J. Phys. Chem. B, 108, 19912 (2004).

[16] C. Berger, Z. Song, X. Li, X. Wu, N. Brown, C. Naud, D. Mayou, T. Li, J. Hass, A. N. Marchenkov, E. H. Conrad, P. N. First, W. A. de Heer; Science, 312, 1191 (2006).

[17] T. Ohta, A. Bostwick, T. Seyller, K. Horn, E. Rotenberg; Science, 313, 951 (2006).

[18] S. Y. Zhou, G. H. Gweon, A. V. Fedorov, P. N. First, W. A. de Heer, D. H. Lee, F. Guinea, A. H. Castro Neto, A. Lanzara; Nature Materials, 6, 770 (2007).

[19] A. L. Vazquez de Parga, F. Calleja, B. Borca, M. C. G. Passeggi Jr, J. J. Hinarejo, F. Guinea, R. Miranda; 
arXiv:0709.0360 [cond-mat].

[20] Y. Ouyang, Y. Yoon, J. Guo; arXiv:0704.2261.

[21] S. Marchini, S. Günther, and J. Wintterlin; Phys. Rev. B, 76, 075429 (2007)

[22] R. Rosei, M. De Crescenzi, F. Sette, C. Quaresima, A. savoia, P. Perfetti; Phys. Rev. B; 28, 1161 (1983).

[23] C. Casiraghi, S. Pisana, K. S. Novoselov, A. K. Geim, A. C. Ferrari, arXiv:0709.2566 1 [cond-mat].

[24] A. C. Ferrari, J. C. Meyer, V. Scardaci, C. Casiraghi, M. Lazzeri, F. Mauri, S. Piscanec, Da Jiang, K. S. Novoselov, S. Roth, A. K. Geim; Phys. Rev. Lett., 97, 187401 (2006).

[25] S. Pisana, M. Lazzeri, C. Casiraghi, K. S. Novoselov, A. K. Geim, A. C. Ferrari, F. Mauri; Nature Materials, 6, 198 (2007).

[26] S. Piscanec, M. Lazzeri, F. Mauri, A. C. Ferrari, J. Robertson; Phys. Rev. Lett., 93, 185503 (2004).

[27] S. Latil, V. Meunier, L. Henrard; Phys. Rev. B, 76, 201402(R) (2007).

[28] C. Casiraghi, A. Hartschuh, E. Lidorikis, H. Qian, H. Harutyunyan, T. Gokus, K. S. Novoselov, A. C. Ferrari; Nano. Lett., 7, 2711 (2007).

[29] P. Blake, E. W. Hill, A. H. Castro Neto, K. S. Novoselov, D. Jiang, R. Yang, T. J. Booth, A. K. Geim; Appl. Phys. Lett., 91, 063124 (2007).

[30] A. C. Ferrari; Solid State Communications, 143, 47 (2007).

[31] A. Das, S. Pisana, S. Piscanec, B. Chakraborty, S. K. Saha, U. V. Waghmare, R. Yang, H. R. Krishnamurhthy, A. K. Geim, A. C. Ferrari, A. K. Sood; arXiv:0709.1174v1.

[32] X. Lu, H. Huang, N. Nemchuk, R. S. Ruoff; Appl. Phys. Lett., 75, 193 (1999).

[33] R. Saito, G. Dresselhaus, M. S. Dresselhaus, Physical Properties of Carbon Nanotubes (Imperial, London, 1998).

[34] S. Reich, C. Thomsen, J. Maultzsch; Carbon Nanotubes: Basic Concepts and Physical Properties (WileyVCH, Berlin, 2004).

[35] K. Nakada, M, Fujita, G. Dresselhaus, M. S. Dresselhaus; Phys. Rev. B, 54, 17954 (1996).

[36] M. Fujita, K. Wakabayashi, K. Nakada, K. Kusakabe; J. of the Phys. Soc. of Japan, 65, 1920 (1996).

[37] Y. Miyamoto, K. Nakada, M. Fujita; Phys. Rev. B, 59, 9858 (1999).

[38] K. Wakabayashi, M. Fujita, H. Ajiki, M. Sigrist; Phys. Rev. B, 59, 8271 (1999).

[39] Y. W. Son, M. L. Cohen, S. G. Louie; Phys. Rev. Lett., 97, 216803 (2006).

[40] L. Pisani, J. A. Chan, B. Montanari, N. M. Harrison; Phys. Rev. B, 75, 064418 (2007).

[41] K. Nakada, M. Igami, M. Fujita; J. of the Phys. Soc. of Japan, 67, 2388 (1998)

[42] Y. Niimi, T. Matsui, H. Kambara, K. Tagami, M. Tsukada, H. Fukuyama; Phys. Rev. B, 73, 085421 (2006).

[43] Y. Kobayashi, K. Fukui, T. Enoki, K. Kusakabe, Y. Kaburagi; Phys. Rev. B, 71, 193406 (2005).

[44] F. Sols, F. Guinea, A. H. Castro Neto; Phys. Rev. Lett., 99, 166803 (2007).

[45] Y. Niimi, T. Matsui, H. Kambara, K. Tagami, M. Tsukada, and H. Fukuyama; Appl. Surf. Sci., 241, 43 (2005).

[46] K. Sasaki, S. Murakami, R. Saito; Appl. Phys. Lett., 88, 113110 (2006).

[47] M. Fujita, M. Igami, K. Nakada; J. of the Phys. Soc. of Japan, 66, 1864 (1997).

[48] S. Okada, A. Oshiyama; Phys. Rev. Lett., 87, 146803
(2001).

[49] H. Lee, Y. W. Son, N. Park, S. Han, J. Yu; Phys. Rev. B, 72, 174431 (2005).

[50] Y. W. Son, M. L. Cohen, S. G. Louie; Nature (London), 444, 347 (2006).

[51] V. Barone, O. Hod, G. E. Scuseria; Nano Lett., 6, 2748 (2006).

[52] H. Zheng, Z. F. Wang, T. Luo, Q. W. Shi, J. Chen; Phys. Rev. B, 75, 165414 (2007).

[53] L. Yang, C. H. Park, Y. W. Son, M. L. Cohen, S. G. Louie; Phys. Rev. Lett. 99, 186801 (2007).

[54] D. Prezzi, D. Varsano, A. Ruini, A. Marini, E. Molinari; arXiv:0706.0916 1 .

[55] O. Hod, V. Barone, J. E. Peralta, G. E. Scuseria; Nano Lett., 7, 2295 (2007).

[56] E. J. Kan, Z. Li, J. Yang, J. G. Hou; arXiv:0708.1213v1.

[57] E. Rudberg, P. Salek, Y. Luo; Nano Lett., 72211 (2007).

[58] O. Hod, V. Barone, G. E. Scuseria; arXiv:0709.0938v2.

[59] I. Martin, Y. M. Blanter; arXiv:0705.0532 v2.

[60] D. A. Areshkin, D. Gunlycke, C. T. White; Nano Lett., 7, 204 (2007).

[61] D. Gunlycke, D. A. Areshkin, C. T. White; Appl. Phys. Lett., 90, 142104 (2007).

[62] T. B. Martins, R. H. Miwa, A. J. R. da Silva, A. Fazzio; Phys. Rev. Lett., 98, 196803 (2007).

[63] B. Huang, F. Liu, J. Wu, B. L. Gu, W. Duan; arXiv:0708.1795 1 .

[64] P. Avouris; Accounts of Chem. Research, 35, 1026 (2002).

[65] P. Avouris, J. Chen; Mater. Today, 9, 46 (2006).

[66] A. P. Graham, G. S. Duesberg, W. Hoenlein, F. Kreupl, M. Liebau, R. Martin, B. Rajasekharan, W. Pamler, R. Seidel, W. Steinhoegl, E. Unger; Appl. Phys. A: Mater. Sci. Process., 80, 1141 (2005).

[67] V. N. Prigodin, N. P. Raju, K. I. Pokhodnya, J. S. Miller, A. J. Epstein; Adv. Mater., 14, 1230 (2002).

[68] R. A. de Groot, F. M. Mueller, P. G. van Engen, K. H. J. Buschow; Phys. Rev. Lett., 50, 2024 (1983).

[69] D. L. Carrol, Ph. Redlich, X. Blase, J. C. Charlier, S. Curran, P. M. Ajayan, S. Roth, M. Rühle; Phys. Rev. Lett., 81, 2332 (1998).

[70] J. Y. Yi, J. Bernholc; Phys. Rev. B, 47, 1708 (1993).

[71] A. H. Nevidomskyy, G. Csányi, M. C. Payne; Phys. Rev. Lett., 91, 105502 (2003).

[72] R. Czerw, M. Terrones, J. C. Charlier, X. Blase, B. Foley, R. Kamalakaran, N. Grobert, H. Terrones, D. Tekleab, P. M. Ajayan, W. Blau, M. Rühle, D. L. Carroll; Nano Lett., 1, 457 (2001).

[73] Y. W. Tan, Y. Zhang, K. Bolotin, Y. Zhao, S. Adam, E.H. Hwang, S. Das Sarma, H. L. Stormer, P. Kim, arXiv:0707.1807 [cond-mat].

[74] A. Morpurgo, private communication.

[75] R. Saito, M. Fujita, G. Dresselhaus, M. S. Dresselhaus; Appl. Phys. Lett., 60, 2204 (1992).

[76] T. W. Odom, J. L. Huang, P. Kim, C. M. Lieber; Nature(London), 391, 62 (1998).

[77] C. D. Spataru, S. Ismail-Beigi, L. X. Benedict, S. G. Louie; Phys. Rev. Lett., 92, 077402 (2004).

[78] B. Özyilmaz, P. Jarillo-Herrero, D. Efetov, P. Kim, arXiv:0709.1731 [cond-mat].

[79] P. L. Giunta, S. P. Kelty; J. Chem. Phys., 114, 1807 (2001).

[80] J. H. Park, E. Vescovo, H. J. Kim, C. Kwon, R. Ramesh, T. Venkatesan; Nature (London), 392, 794 (1998).

[81] S. A. Wolf, D. D. Awschalom, R. A. Buhrman, J. M. 
Daughton, S. von Molnár, M. L. Roukes, A. Y. Chtchelkanova, D. M. Treger; Science, 294, 1488 (2001).

[82] C. M. Fang, G. A. de Wijs, R. A. de Groot; J. Appl. Phys., 91, 8340 (2002).

[83] A. Hirsch; Angew. Chem. Int. Ed., 41, 1853 (2002).

[84] V. Georgakilas, K. Kordatos, M. Prato, D. M. Guldi, M. Holzinger, A. Hirsch; J. Am. Chem. Soc., 124, 760 (2002).

[85] Y.S. Lee, M. B. Nardelli, N. Marzari; Phys. Rev. Lett. 95, 076804 (2005).

[86] J. Kong, N. R. Franklin, C. W. Zhou, M. G. Chapline, S. Peng, K. J. Cho, H. J. Dai; Science, 287, 622 (2000).

[87] H. J. Dai, E. W. Wong, C. M. Lieber; Science, 272, 523 (1996)

[88] A. Star, J. F. Stoddart, D. Steuerman, M. Diehl, A. Boukai, E. W. Wong, X. Yang, S. W. Chung, H. Choi, J. R. Heath; Angew. Chem. Int. Ed., 40, 1721 (2001).

[89] V. Scardaci, A. G. Rozhin, F. Hennrich, W. I. Milne, A. C. Ferrari; Physica E, 37, 115 (2007).

[90] T. Hasan, V. Scardaci, P. Tan, A. G. Rozhin, W. I. Milne, A. C. Ferrari; J. Phys. Chem. C, 111, 12594 (2007)

[91] Z. Xu, Q. S. Zheng, G. Chen; Appl. Phys. Lett., 90, 223115 (2007).

[92] K. Wakabayashi; Phys. Rev. B, 64, 125428 (2007).

[93] F. Muñoz-Rojas, J. Fernández-Rossier, L. Brey, J. J. Palacios; arXiv:0707.0375v1.

[94] Q. Yan, B. Huang, J. Yu, F. Zheng, J. Zang, J. Wu, B. L. Gu, F. Liu, W. Duan; Nano Lett., 7, 1469 (2007).

[95] Z. F. Wang, Q. Li, H. Zheng, H. Ren, H. Su, Q. W. Shi, J. Chen; Phys. Rev. B, 75, 113406 (2007).

[96] Y. Miura, H. Kasai, W. Diño, H. Nakanishi, T. Sugimoto; J. Appl. Phys., 93, 3395 (2003).

[97] T. Roman, W. A. Diño, H. Nakanishi, H. Kasai, T. Sugimoto, K. Tange; Jap. J. Appl. Phys., 45, 1765 (2006).

[98] C. P. Chang, B. R. Wu, R. B. Chen, M. F. Lin; J. Appl. Phys., 101, 063506 (2007).

[99] D. Jiang, B. G. Sumpter, S. Dai; J. of Chem Phys., 126, 134701 (2007).

[100] S. Gowtham, R. H. Scheicher, R. Ahuja, R. Pandey, S.
P. Karna; Phys. Rev. B, 76, 033401 (2007).

[101] K. Kusakabe, M. Maruyama; Phys. Rev. B, 67, 092406 (2003).

[102] G. Cantele, Y. S. Lee, D. Ninno, N. Marzari; V26.00009, APS March Meeting (2007).

[103] M. Wimmer, I. Adagideli, S. Berber, D. Tománek, K. Richter; arXiv:0709.3244

[104] B. Huang, Q. Yan, G. Zhou, J. Wu, F. Liu, B. L. Gu, W. Duan; arXiv:0711.0787

[105] R. Ramprasad, P. von Allmen, L. R. C. Fonseca, Phys. Rev. B, 60, 6023 (1999).

[106] D. Luxembourg, X. Py, A. Didion, R. Gadiou, C. VixGuterl, G. Flamant; Micropor. Mesopor. Mat., 98, 123 (2007).

[107] J. M. Carlsson, M. Scheffler; Phys. Rev. Lett., 96, 046806 (2006).

[108] F. Schedin, A. K. Geim, S. V. Morozov, E. H. Hill, P. Blake, K. S. Novoselov; Nature Materials, 6, 652 (2007).

[109] S. J. Clark, M. D. Segall, C. J. Pickard, P. J. Hasnip, M. J. Probert, K. Refson, M. C. Payne; Zeitschrift für Krystallographie, 220, 567 (2005).

[110] J. P. Perdew, K. Burke, M. Ernzerhof; Phys. Rev. Lett., 77, 3865 (1996).

[111] D. Vanderbilt; Phys. Rev. B, 41, 7892 (1990).

[112] M. Avriel; Nonlinear Programming: Analysis and Methods (2003), Dover Publishing.

[113] H. J. Monkhorst, J. D. Pack; Phys. Rev. B, 13, 5188 (1976).

[114] T. O. Wehling, K. S. Novoselov, S. V. Morozov, E. E. Vdovin, M. I. Katsnelson, A. K. Geim, A. I. Lichtenstein; arXiv:cond-mat/0703390

[115] O. Leenaerts, B. Partoens, F. M. Peeters; arXiv:0710.1757 1 .

[116] B. Uchoa, C. Y. Lin, A. H. Castro Neto; arXiv:0706.1237 2 .

[117] I. Zanella S. Guerini, S. B. Fagan, J. Mendes Filho, A. G. Souza Filho; arXiv:0711.1131. 